\title{
DISTRIBUTION OF RHEUMATIC DISEASES IN A REFERRAL CENTER IN BAHIA/BRAZIL
}

Adriane Souza da Paz ${ }^{1}$, Mittermayer Barreto Santiago ${ }^{1}$

1.Universidade Federal da Bahia, Salvador (BA), Brazil.

${ }^{\star}$ Corresponding author: adriane.spaz@hotmail.com

\section{BACKGROUND}

Epidemiological data on rheumatological diseases in Brazil are scarce, but such studies should be encouraged by health managers for adequate planning of actions in this area. The prevalence of each disease depends on the geographic region, genetic, and environmental factors, and may vary from one service to another. The objective of the present study was to evaluate the profile of patients followed-up at a referral university rheumatological unit specialized in the treatment of diffuse connective tissue diseases in the state of Bahia.

\section{MATERIALS AND METHODS}

This is a retrospective study of the medical records of patients registered performed with 2183 patients and followed-up at the Rheumatology Service of Professor Edgard Santos University Hospital during the year of 2019 in Salvador, Bahia. Electronic medical records were systematically evaluated by one of the authors, and the diagnosis based on the classification criteria of different rheumatological diseases.

\section{RESULTS}

A total of 2135 medical records was evaluated, being 1816 females (85\%) and 319 males (15\%), median age 49 years (range 18-91 years). The main diseases in order of frequency were: systemic lupus erythematosus (810), rheumatoid arthritis (466), scleroderma (103), spondyloarthritis (91), overlap syndrome (89), chikungunya arthritis (85), dermatomyositis and polymyositis (47), psoriatic arthritis (42), Takayasu arteritis (37) and primary Sjögren's syndrome (36).

\section{CONCLUSION}

The epidemiological profile observed in the present study reveals a higher prevalence of systemic lupus erythematosus and rheumatoid arthritis. It is justified as our institution is a tertiary referral center in the treatment of highly complex rheumatological diseases and differs from that observed in the majority of other services. Of note, the significant number of cases of chikungunya arthritis reflects the present outbreak of this infection in our state. 